\title{
CONFORMAL GEOMETRY OF RICCI FLAT 4-MANIFOLDS
}

Dedicated to Professor Shoshichi Kobayashi on his sixtieth birthday

\author{
By Mitsuhiro ITOH
}

\section{Introduction}

Let $M$ be a compact, connected oriented 4-manifold. Let $g$ be a smooth Riemannian metric on $X$. Then the Riemannian curvature tensor $R=R_{\imath j k l}$ is defined naturally, and the Ricci tensor $R i c=R_{\imath}$ and the scalar curvature $R_{g}$ by the trace of the Riemannian curvature tensor and the trace of the Ricci tensor, respectively.

Further we define the Weyl conformal tensor $W=W_{\imath j k l}$ by a linear combination of $R=R_{\imath j k l}$, Ric and $R_{g}$ in such a way that the tensor $W$ is invariant under a conformal change of metrics.

We will investigate in this paper the moduli $\mathcal{E}(M)$ of Ricci flat metrics on certain 4-manifolds $M$ from 4-dimensional conformal geometry. Here we mean by the moduli the space of all Ricci flat metrics of volume one modulo diffeomorphisms of $M$.

Since a Ricci flat metric is Einstein, the moduli is considered naturally as the moduli of Einstein metrics of $R_{g}=0$.

We have indeed the following premoduli theorem due to K. Koiso ([16] and $[5])$.

Given an Einstein metric $g$. Then there is a finite dimensional real analytic submanifold $\mathscr{Z}$ in a slice $\mathcal{S}$ at $g$ such that (i) $g \in \mathscr{L}$, (ii) $T_{g} \mathscr{L}$ coincides with the space of infinitesimal Einstein deformations and (iii) the intersection $\mathcal{E}(M) \cap \mathcal{S}$, called the premoduli around $g$, is a real analytic subvariety of $\mathcal{Z}$.

We restrict ourself to Ricci flat 4-manifolds having topological invariant $\chi+(3 / 2) \tau=0$, more precisely, manifolds whose universal covering is a K3 surface. We can then apply the Torelli type theorem for K3 surfaces together with the notion of anti-self-dual conformal structure and get a complete description of manifold structure of $\mathcal{E}(M)$.

By applying the Chern-Weil theorem for characteristic classes one has the following identity which is valid for an arbitrary Riemannian 4-manifold $(M, g)$

$$
\chi(M)+\frac{3}{2} \tau(M)=\frac{1}{48 \pi^{2}} \int_{M}\left\{R_{g}^{2}-3|R i c(g)|^{2}\right\} d v_{g}+\frac{1}{4 \pi^{2}} \int_{M}\left|W^{+}(g)\right|^{2} d v_{g}
$$

Received November 12, 1993. 
Here $\chi(M)=\sum_{k}(-1)^{k} b_{k}(M)$ is the Euler number and $\tau(M)$ is the index $b^{+}(M)-$ $b^{-}(M)$ of the cup product $q_{M}: H^{2}(M, \boldsymbol{Z}) \times H^{2}(M, \boldsymbol{Z}) \rightarrow H^{4}(M, \boldsymbol{Z}) \cong \boldsymbol{Z}$ and $W^{+}$denotes the self-dual Weyl conformal tensor, i. e., $W^{+} \in \Gamma\left(M, \Omega^{+} \otimes \Omega^{+}\right)\left(\Omega^{+}=\Omega_{g}^{+}\right.$is the self-dual 2 -form bundle of $M$ ).

From this we observe the following

Proposition 0.1. Let $M$ be of $\chi(M)+(3 / 2) \tau(M)=0$.

Let $g$ be a metric on $M$ satisfying $W^{+}=0$. Then the scalar curvature $R_{g}=0$ if and only if $g$ is Ricci flat.

For a 4-manifold $M$ satisfying $(\chi+(3 / 2) \tau)(M)=0$ the moduli $\mathcal{E}(M)$ of Ricci flat metrics on $M$ coincides from this proposition with the quotient space of metrics satisfying the equations $W^{+}(g)=0, R_{g}=0$ modulo the diffeomorphism action.

Since the equation $W^{+}=0$ as a section of $\Omega^{+} \otimes \Omega^{+}$and the sign of constant scalar curvature are conformal invariant, the latter space is regarded as the moduli of anti-self-dual conformal structures of zero scalar curvature, which we denote by $\mathscr{M}^{(0)}(M)$.

Metrics $g$ and $g_{1}$ are said to be conformally equivalent if $g_{1}=f g$ for a positive function $f$ and then the conformal equivalence defines a conformal structure $[g]$ represented by $g$. We say a conformal structure $[g]$ to be antiself-dual if $W^{+}(g)=0$ and we define the moduli $\mathscr{M}(M)$ of anti-self-dual conformal structures on $M$ to be the space of all anti-self-dual conformal structures on $M$ modulo the diffeomorphism action so that we have

$$
\mathcal{E}(M) \cong \mathscr{M}^{(0)}(M) \subset \mathscr{M}(M) .
$$

From the 4-dimensional speciality, for any $(M, g)$ having $W^{+}=0$ there exists an elliptic complex which provides the moduli $\mathscr{M}(M)$ a real analytic variety structure.

In fact, the following sequence enjoys the ellipticity

$$
0 \longrightarrow \Gamma(M, T) \longrightarrow \Gamma\left(M, S_{0}^{2}\left(T^{*}\right)\right) \longrightarrow \Gamma\left(M, S_{0}^{2}\left(\Omega^{+}\right)\right) \longrightarrow 0
$$

with cohomology groups $\boldsymbol{H}^{0}, \boldsymbol{H}^{1}, \boldsymbol{H}^{2}$ of finite dimension so that one gets a real analytic variety theorem in terms of these cohomology groups ([11]).

The following theorem gives the characterization of Ricci flat 4-manifolds of $\chi+(3 / 2) \tau=0$.

THEOREM $0.2([8])$. Let $(M, g)$ be a compact, connected oriented Riemannian 4-manifold.

(i) If $(M, g)$ is Einstein, then $\chi(M)+(3 / 2) \tau(M) \geqq 0$ and the equality $\chi(M)+$ $(3 / 2) \tau(M)=0$ holds if and only if $g$ is Ricci flat and anti-self-dual (i.e., $\left.W^{+}=0\right)$.

(ii) If $(M, g)$ is Ricci flat and $W^{+}=0$ (so that $\left.\chi+(3 / 2) \tau=0\right)$, then, either (a) $(M, g)$ is flat and is covered by a flat Riemannian 4-torus $T^{4}$ or (b) $(M, g)$ is a Kähler Einstein $K 3$ surface $\left(\pi_{1}=1\right)$, a Kähler Einstein Enriques surface $\left(\pi_{1}=Z_{2}\right)$ 
or the quotient of a Kähler Einstein Enriques surface by a free anti-holomorphic isometric involution $\left(\pi_{1}=\boldsymbol{Z}_{2} \times \boldsymbol{Z}_{2}\right)$.

To state our theorems we begin with a technical preliminary in terms of groups of diffeomorphisms.

We denote by $\operatorname{Diff}^{+}=\operatorname{Diff}^{+}(M)$ the group of orientation preserving diffeomorphisms of $M$. We denote moreover by $\operatorname{Diff}^{\prime}=\operatorname{Diff}^{\prime}(M)$ and $\operatorname{Diff}^{o}=\operatorname{Diff}^{\circ}(M)$ the normal subgroups of $\operatorname{Diff}^{+}\left\{\phi \in \mathrm{Diff}^{+} ; \phi^{*}=\mathrm{id}\right.$ on $\left.H^{2}(M, Z)\right\}$ and $\left\{\phi \in \mathrm{Diff}^{+}\right.$; $\phi \cong \mathrm{id}_{M}$, isotopic $\}$, respectively. Here two diffeomorphisms $\phi$ and $\phi_{1}$ are said to be isotopic, if there exists a path in Diff ${ }^{+}$joining $\phi$ and $\phi_{1}$.

So $\operatorname{Diff}^{\circ} \subset$ Diff $^{\prime} \subset$ Diff $^{+}$and accordingly we have $\hat{\mathcal{E}}(M), \tilde{\mathcal{E}}(M)$ and $\mathcal{E}(M)$, the isotopy-Teichmüller moduli, the Teichmüller moduli and the modul, respectively.

There are canonical projections

$$
\hat{\mathcal{E}}(M) \longrightarrow \tilde{\mathcal{E}}(M) \longrightarrow \mathcal{E}(M)
$$

such that $\mathcal{E}(M)=\tilde{\mathcal{E}}(M) / \Gamma(M)$ and $\tilde{\mathcal{E}}(M)=\hat{\mathcal{E}}(M) / \Gamma^{\prime}(M)$ where $\Gamma^{\prime}(M)=\mathrm{Diff}^{+} / \mathrm{Diff}^{\prime}$ and $\Gamma^{\prime}(M)=$ Diff $^{\prime} /$ Diff $^{\circ}$ are the mapping class groups of $M$.

A K3 surface is the 4-manifold of which the moduli $\mathcal{E}$ is completely well studied. Actually we have the so-called period map and the Torelli type theorem (see [K, $\S 12$ in 5] or [14] for the details and we will give a brief argument of this theorem in $\S 1$ ).

THEOREM 0.3 (Torelli type Theorem). The Teichmüller moduli $\tilde{\varepsilon}$ of Ricci flat metrics on a K3 surface $X$ is isomorphic to an open dense subset $T$ of $G r_{3,22}^{+}$ $=S O(3,19) / S O(3) \times S O(19)$. The modul $\mathcal{E}$ is then isomorphic to the quotient of $T$ modulo the mapping class group $\Gamma(X)=\mathrm{Diff}^{+} / \mathrm{Diff}^{\prime}$.

The space $S O(3,19) / S O(3) \times S O(19)$ is an irreducible symmetric space of non-compact type whose dimension is 57 .

The period map pe: $\tilde{\mathcal{E}} \rightarrow G r_{3,22}^{+}$is the assignment of the naturally oriented $H^{+}(g) \subset H^{2}(X, \boldsymbol{R})$ to each Ricci flat metric $g$ and the Torelli type theorem asserts that $p e$ gives the embedding of the Teichmüller moduli into the Grassmannian manifold and exactly onto $T\left(H^{+}(g)\right.$ is the space of self-dual harmonic 2-forms on $\left.X, \operatorname{dim}_{R} H^{+}(g)=3\right)$.

It follows from this theorem that the moduli $\varepsilon$ for a $\mathrm{K} 3$ surface $X$ is a locally symmetric orbifold. In fact the moduli carries finite group quotient singuralities, since the isometry group $I(g)$ of $g$ is a finite group which is isomorphic to the isotropy at $p e(g) \in T$ in the group $\operatorname{Aut}\left(H^{2}(X, Z), q_{X}\right) \cap S O(3,19)$ and $I(g)$ varies when $g$ moves (see [5]).

On the other hand, it is not difficult to show that the action of $\Gamma^{\prime}(X)$ on $\hat{\mathcal{E}}(X)$ is free (see Proposition 2.5) so that the isotopy-Teichmüller moduli $\hat{\mathcal{E}}$ for a 4-manifold underlying a $\mathrm{K} 3$ surface is a smooth manifold (possibly having many components) of which each component is isometric to $\tilde{\mathcal{E}}$ (see Theorem 
2.6). The number of components is the order of $\Gamma^{\prime}(X)=\operatorname{Diff}^{\prime}(X) / \operatorname{Diff}^{\circ}(X)$.

By Theorem 0.2 an Enriques surface $Y$ and a 4 -manifold $Z$, a $Z_{2}$-quotient of $Y$, are written as quotients of a $\mathrm{K} 3$ surface $X$ modulo the respective covering transformation groups $\Sigma . \quad \Sigma \subset \operatorname{Diff}^{+}(X)$ induces isometric transformations of the isotopy-Teichmüller moduli for a $\mathrm{K} 3$ manifold $X$. Here isometries mean transformations of the moduli preserving the "canonically equipped $L^{2}$ metric". It follows then that the fixedpoint set of the action of $\Sigma$, which is totally geodesic as a submanifold in the moduli for $X$, provides the manifold structures to the isotopy-Teichmüller moduli for 4-manifolds $Y$ and $Z$.

THEOREM 0.4. Let $Y$ be a 4-manifold underlying an Enriques surface. Then the isotopy-Teichmüller moduli $\hat{\mathcal{E}}(Y)$ is isometrically embedded in $\hat{\mathcal{E}}(X)$ as a 29 dim totally geodesic submanifold. Each component of this totally geodesic submanifold is via the period map isometric to a submanifold $T \cap F$ in $T$ where $F$ is the fixedpoint set of the involutive deck transformation, written in the form

$$
G r_{1,10}^{+} \times G r_{2,12}^{+}=(S O(1,9) / S O(1) \times S O(9)) \times(S O(2,10) / S O(2) \times S O(10)),
$$

embedded in $S O(3,19) / S O(3) \times S O(19)$.

For a $Z_{2}$-quotient of an Enriques surface we have similarly

THEOREM 0.5. Let $Z$ be a $Z_{2}$-quotient of an Enrqques surface. Then the isotopy-Teichmüller moduli $\hat{\varepsilon}(Z)$ is embedded in $T \subset S O(3,19) / S O(3) \times S O(19)$ isometrically and totally geodesically. This totally geodesic submanifold is 15 dimensional.

From Theorems 0.4 and 0.5 we have

COROLlary 0.6. Let $Y$ and $Z$ be an Enriques surface and its anti-holomorphic $\boldsymbol{Z}_{2}$-quotient, respectively. Then the Teichmüller moduli $\tilde{\mathcal{E}}$ and the moduli $\mathcal{E}$ of Ricci flat metrics on $Y$ or on $Z$ admit at least an orbifold structure.

It is open whether $\tilde{\mathcal{E}}$ for $Y$ and $Z$ is smooth, or equivalently whether the mapping class group Diff $/$ Diff $^{o}$ acts freely on the isotopy-Teichmüller moduli $\hat{\mathcal{E}}$.

\section{$\S 1$. The moduli of Ricci flat metrics on a K3 manifold}

A K3 surface is, by definition, a compact, connected complex surface with trivial canonical line bundle and $b^{1}=0$ (for references of K3 surfaces see [§ VIII, 4]).

We say a compact 4-manifold which underlines a $\mathrm{K} 3$ surface as a $\mathrm{K} 3$ manifold.

Example 1. Let $\iota: T^{2} \rightarrow T^{2} ;\left(z_{\imath}\right) \rightarrow\left(-z_{\imath}\right)$ be the involution defined on a 2-dim 
complex torus $T^{2}$. The quotient $T^{2} / \iota$ has sixteen singular points. By blowing up these points we get a smooth surface, called a Kummer surface, which is simply connected and the canonical line bundle $K$ is trivial as a holomorphic line bundle.

Example 2. A hypersurface of degree 4 in $\boldsymbol{P}^{3}(C)$ is simply connected and has the first Chern class $c_{1}=0$. So this complex surface is a K3 surface.

The following theorem is classical.

THEOREM 1.1. (i) Any K3 surfaces are diffeomorphic.

(ii) ([18]) Every K3 surface has a Kähler metric.

(iii) ([20]) Every K3 surface admits a Ricci flat Kähler metric.

A K3 manifold $X$ has the topological invariants; $\chi=24, b_{2}=22$ and $\tau=-16$ so that $b^{+}=3, b^{-}=19$ where $\left(b^{+}, b^{-}\right)$is the signature of the cup product of $H^{2}(X ; \boldsymbol{Z})$.

Consider the moduli $\hat{\mathcal{E}}, \tilde{\mathcal{E}}$ and $\mathcal{E}$ of Ricci flat metrics on $X$.

TheOREM 1.2 (Torelli type Theorem). Via the period map, the Teichmüller moduli $\tilde{\mathcal{E}}(X)$ is isomorphic to an open dense subset $T$ of $S O(3,19) / S O(3) \times S O(19)$.

To define the period map and explain the domain $T$ we need to state the cup product more precisely.

The cup product $q_{X}$ of a K3 manifold $X$ has the form $q_{X} \cong \oplus^{2}\left(-E_{8}\right) \oplus \oplus^{3} H$ where $E_{8}$ is the Cartan matrix of type $E_{8}$ and $H$ is $\left(\begin{array}{ll}0 & 1 \\ 1 & 0\end{array}\right)$.

Let $g$ be a metric on $X$. Then from the harmonic theory the space $H^{2}(X, g)$ of $g$-harmonic 2 -forms on $X$, which is isomorphic to the second cohomology group $H^{2}(X ; \boldsymbol{R})$, decomposes as the sum of the spaces $H^{+}(X, g)$ of self-dual harmonic 2 -forms and anti-self-dual harmonic 2 -forms;

$$
H^{2}(X, g)=H^{+}(X, g) \oplus H^{-}(X, g)
$$

for which $q_{X}>0$ on $H^{+}(X, g)$ and $q_{X}<0$ on $H^{-}(X, g)$.

$H^{+}(g)$ and $H^{-}(g)$ are orthogonal with respect to $q_{X}$. Here we identify $q_{X}$ on $H^{2}(X, \boldsymbol{R})$ with the wedge product on deRham cohomology classes of closed 2 -forms.

Now suppose the metric $g$ is Ricci flat.

Then from Proposition 0.1 this metric is anti-self-dual and of zero scalar curvature. So any harmonic self-dual 2 -form must be parallel by the Weitzenböck-Bochner argument and induces up to a constant a complex structure $J$ on $X$ compatible with $g$. The metric $g$ is Ricci flat Kähler with respect to the complex surface $(X, J)$.

We can assign from this fact a canonical orientation to $\mathrm{H}^{+}(X, g)$. 
In fact, if we choose $\alpha, \beta \in H^{+}(g), \alpha \neq \beta$, to which complex structures $J_{\alpha}$, $J_{\beta}$ associate, then we have another $\gamma \in H^{+}(g)$ such that $J_{\gamma}=J_{\alpha^{\circ}} J_{\beta}$ and hence the orientation $\{\alpha, \beta, \gamma\}$ for $H^{+}(g)$.

We therefore get the assignment

$$
g \bmod \text { Diff }^{\prime} \longmapsto \text { oriented } \operatorname{pr}\left(H^{+}(g)\right)
$$

which yields the period map

$$
p e: \tilde{\mathcal{E}}(X) \longrightarrow G r_{3,22}^{+} \cong S O(3,19) / S O(3) \times S O(19) .
$$

Here we regard first $H^{+}(g)$ as a 3-dim linear subspace of the infinite dimensional vector space $Z^{2}(X)$, the space of all closed 2-forms on $X$ and then take $\operatorname{pr}\left(H^{+}(g)\right)$, namely the deRham cohomology projection of $H^{+}(g)$ in $H^{2}(X, \boldsymbol{R})$, as a subspace in the 22-dim space $H^{2}(X, \boldsymbol{R})$.

The subset $T$ is defined as follows.

Let $\Delta=\left\{\gamma \in H^{2}(X ; \boldsymbol{Z}) ; q_{X}(\gamma, \gamma)=-2\right\}$ be the set of "roots" and, for any oriented positive 3-plane $\Pi$ in $H^{2}(X ; \boldsymbol{R})$ we denote by $\Pi^{\perp}$ the $q_{X}$-orthogonal complement of $\Pi ; \Pi^{\perp}=\left\{\alpha \in H^{2}(X ; \boldsymbol{R}) ; q_{X}(\alpha, \Pi)=0\right\}$. Then $T$ is the set of all oriented positive 3-planes $\Pi \subset H^{2}(X ; \boldsymbol{R})$ such that $\Pi^{\perp} \cap \Delta=\emptyset . \quad T$ is the complement of countably many unions of codimension 3 submanifolds in $G r_{3,22}^{+}$. So, $T$ is connected and simply connected.

The reason why one excludes the complement of $T$ from $G r_{3,22}^{+}$stems from the fact that $q_{X}$ is even and the canonical line bundle is trivial so that an arbitrary complex curve $C$ in a K3 surface satisfies $C \cdot C \geqq-2$ and if $C \cdot C \neq-2$, then $C \cdot C \geqq 0([5]$, [13]).

We omit proving that the period map is isomorphic. See [5] for the references and see also references cited there.

The space $S O(3,19) / S O(3) \times S O(19)$ is the noncompact dual of an ordinary Grassmannian manifold $G r_{3,22}$. So it is a symmetric space of noncompact type and has the invariant metric. At the origin this invariant metric $\langle$,$\rangle is given$ by the restriction of the negative Killing form of the Lie algebra $\mathfrak{g} \mathfrak{D}(3,19)$. Actually the tangent space at the orgin $T_{o}$ is identified with $\mathfrak{m}=\{3 \times 19$ matrices $\}$ and the invariant metric is, up to constant,

$$
\langle U, V\rangle=\operatorname{tr}\left(U V^{t}+V U^{t}\right), \quad U, V \in \mathfrak{m} .
$$

We interpret this invariant metric in terms of $\operatorname{Hom}\left(\mathrm{H}^{+}, \mathrm{H}^{-}\right)$as follows.

Take a Ricci flat metric $g$ corresponding to the origin $o$. Then $T_{o} \cong \mathfrak{m}$ is identified with the space of homomorphisms; $H^{+}(g) \rightarrow H^{-}(g)$. For a homomorphism $f: H^{+} \rightarrow H^{-}$we denote by $f^{*}: H^{-} \rightarrow H^{+}$the adjoint of $f$, namely $q_{X}(f(\alpha), \beta)$ $=q_{X}\left(\alpha, f^{*}(\beta)\right), \alpha \in H^{+}$and $\beta \in H^{-}$.

Then the invariant metric $\langle$,$\rangle has the form$

$$
\left\langle f, f_{1}\right\rangle=-\operatorname{tr}\left(f f_{1}^{*}+f_{1} f^{*}\right), \quad f, f_{1} \in H^{+} .
$$




\section{§ 2. Anti-self-dual conformal structures on a K3 manifold}

We will look at the moduli $\hat{\mathcal{E}}(X)$ and $\tilde{\varepsilon}(X)$ by means of anti-self-dual conformal structures.

Before doing we prepare the notion of anti-self-dual conformal structure and define several kinds of moduli of anti-self-dual conformal structures on $X$.

Suppose $M$ is a compact, connected oriented 4-manifold. Let $g$ be a metric on $M$. Then the Weyl conformal tensor $W$ of $g$ gives a section of the bundle $S_{o}^{2}\left(\Omega^{2}\right)$, the tracefree symmetric product of $\Omega^{2}$. This is because $W_{\imath j k l}=-W_{j i k l}$ $=W_{k l \imath}$ and $\sum_{i k} g^{i k} W_{\imath j k l}=0$.

Decomposing $\Omega^{2}$ into the sum as $\Omega^{2}=\Omega^{+} \oplus \Omega^{-}$for the self-dual 2-form bundle $\Omega^{+}$and the anti-self-dual 2 -form bundle $\Omega^{-}$, we have the decomposition of $W$ as $W=\left(W^{+}, W^{-}\right)$where $W^{+} \in \Gamma\left(M, S_{o}^{2}\left(\Omega^{+}\right)\right), W^{-} \in \Gamma\left(M, S_{o}^{2}\left(\Omega^{-}\right)\right)$and the $\Omega^{+} \otimes \Omega^{-}$component of $W$ vanishes.

Definition ([2]). A metric $g$ on $M$ is called anti-self-dual if the $S_{o}^{2}\left(\Omega^{+}\right)$component $W^{+}=0$.

We say that a conformal structure $[g]$ represented by $g$ is anti-self-dual if $W^{+}(g)=0$.

Let $\phi$ be an orientation preserving diffeomorphism of $M$. Then the pull back metric $\phi^{*} g$ by $\phi$ defines a conformal structure $\left[\phi^{*} g\right]$ which we denote by $\phi^{*}[g]$ so that $\phi$ gives a transformation of $\mathcal{C}(M)$ where $\mathcal{C}(M)$ is the space of all conformal structures on $M$.

Since the self-dual Weyl conformal tensor $W^{+}$of $\phi^{*} g$ is just the pull back of $W^{+}$of $g$ by $\phi$, the space $\mathcal{C}^{-}(M)$ of all anti-self-dual conformal structures on $M$ is invariant under the action of arbitrary $\phi \in \operatorname{Diff}^{+}(M)$. So we can get the quotient spaces $\mathscr{M}(M)=\mathcal{C}^{-}(M) / \operatorname{Diff}^{+}, \tilde{M}(M)=\mathcal{C}^{-}(M) / \operatorname{Diff}^{\prime}$ and $\mathscr{M}(M)=\mathcal{C}^{-}(M) /$ Diff $^{o}$ and we call them the moduli, the Teichmüller moduli and the isotopy-Teichmüller moduli, respectively, of anti-self-dual conformal structures on $M$.

Let $g$ be an anti-self-dual metric. Then it induces the following elliptic complex ;

$$
0 \longrightarrow \Gamma(M, T) \stackrel{L_{g}}{\longrightarrow} \Gamma\left(M, S_{o}^{2}\left(T^{*}\right)\right) \stackrel{D_{g}}{\longrightarrow} \Gamma\left(M, S_{o}^{2}\left(\Omega^{+}\right)\right) \longrightarrow 0
$$

Here $T, T^{*}$ are the tangent, cotangent bundles and $L=L_{g}$ is the tracefree Lie derivative of vector fields on $X ;(L(U))_{i j}=\nabla_{i} U_{j}+\nabla_{j} U_{i}-1 / 2\left(\sum_{k} \nabla_{k} U_{k}\right) g_{\imath \jmath}$. Further $D=D_{g}$ is the Fréchet derivative of the self-dual Weyl conformal tensor $W^{+}$; $D(h)=d /\left.d t\right|_{t=0} W^{+}\left(g_{t}\right)$ for $h=d /\left.d t\right|_{t=0} g_{t}$.

The cohomology spaces are $\boldsymbol{H}^{0}=\{U \in \Gamma(T), L(U)=0\}, \boldsymbol{H}^{1}=\left\{h \in \Gamma\left(S_{o}^{2}\left(T^{*}\right)\right)\right.$; $\left.D(h)=0, L^{*}(h)=0\right\}$ and $\boldsymbol{H}^{2}=\left\{B \in \Gamma\left(S_{o}^{2}\left(\Omega^{+}\right)\right), D^{*} B=0\right\}$.

The index of this elliptic complex is from the Atiyah-Singer index theorem $h^{0}-h^{1}+h^{2}=1 / 2(29 \tau(M)+15 \chi(M))\left(h^{\imath}=\operatorname{dim} \boldsymbol{H}^{i}, \imath=1,2,3\right)$. 
Remark. Any diffeomorphism $\phi \in \operatorname{Diff}^{+}(M)$ induces an isomorphism of elliptic compleces

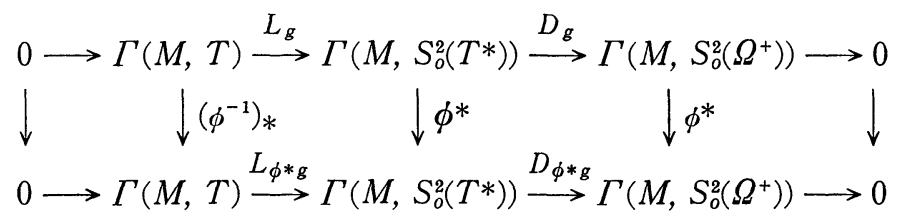

so that if $\phi$ is an isometry of an anti-self-dual metric $g$, then $\phi$ induces an isomorphism of $\boldsymbol{H}^{i}, i=0,1,2$.

Now consider a Ricci flat metric $g$ on a K3 manifold $X$. From Proposition $0.1 \mathrm{~g}$ is anti-self-dual and has zero scalar curvature.

Proposition 2.1. Let $g$ be a Ricci flat metric on a $K 3$ manifold $X$. Then the cohomology groups are $\boldsymbol{H}^{0}=0, \boldsymbol{H}^{1} \cong \boldsymbol{R}^{57}$ and $\boldsymbol{H}^{2} \cong \boldsymbol{R}^{5}$.

Proof. $\quad \boldsymbol{H}^{0}$ is the space of conformal Killing fields. First we show that the group of conformal transformations for the conformal structure [g] coincides with the group of isometries for $g$. Since $g$ is Ricci flat, it is a Yamabe metric of Yamabe invariant zero for the conformal structure [g]. From [9] the uniqueness of Yamabe metrics of nonpositive Yamabe invariant applies so that an arbitrary conformal transformation for $[g]$ is an isometry for $g$. Since conformal transformations generated by a conformal Killing field $U$ are isometries, $U$ is a Killing field. Because of the Ricci flatness the 1-form $\xi$ corresponding to $U$ must be parallel (by Theorem 2.3 in [15], for example) and hence $\xi$ vanishes from $b_{1}=0$. So $\boldsymbol{H}^{0}=0$.

To compute $\operatorname{dim} \boldsymbol{H}^{2}$ we can apply the Weitzenböck-Bochner formula given in [12] to the elliptic operator of fourth order $D^{*} D$.

Actually, since $g$ is Ricci flat, $D^{*} D B=\left(\nabla^{*} \nabla\right)^{2} B$ for $B \in \Gamma\left(S_{o}^{2}\left(\Omega^{+}\right)\right)$, where $\nabla^{*} \nabla$ is the covariant Laplacian, so that $B \in \boldsymbol{H}^{2}$ if and only if $B$ is a parallel section of the bundle $S_{o}^{2}\left(\Omega^{+}\right)$. Thus $h^{2}=5$. Here we used the fact that for a K3 manifold $X$ the orthonormal basis of $H^{+}(g)$ makes the bundle $\Omega^{+}$trivial and then $S_{o}^{2}\left(\Omega^{+}\right)$has five, linearly independent parallel sections.

The index of the complex for $X$ is -52 so that $\operatorname{dim} \boldsymbol{H}^{1}=57$.

Now we state the real analytic variety theorem for the isotopy-Teichmüller moduli $\hat{\mathscr{M}}(M)$ valid for a general 4 -manifold $M$ except for $S^{4}$.

THEOREM 2.2. Let $M$ be a compact, connected oriented 4-manifold and $g$ an anti-self-dual metric on $M$. Let $[g]$ be the corresponding point in $\hat{M}(M)$ (here we identify the conformal structure $[g]$ and the point of the moduli derived from $[g]$ by the diffeomorphism quotient). Then $\hat{\mathcal{M}}(M)$ is isomorphic around $[g]$ to the real analytic variety $\left\{h \in \boldsymbol{H}^{1} ;|h|<\varepsilon, \Psi(h)=0\right\} / C_{g}^{o}$, where $\Psi: \boldsymbol{H}^{1} \rightarrow \boldsymbol{H}^{2}$ is an analytic map associated to the Kuranishi map, $\Psi(0)=0$ and $C_{g}^{o}=C_{g} \cap$ Diffo $^{o}$ denotes 
the group of conformal transformations of $[g]$.

See (iv), Sect. 3 in [11] for the details. We explain here the action $C_{g}^{o}$ on the complex (2.1). We noticed in the remark that any $\phi \in \operatorname{Diff}^{+}(M)$ acts on (2.1) equivariantly. Since $C_{g}^{o}$ is compact except for the standard 4-sphere (for instance see [9]), we can choose a metric $g$ inside the conformal structure $[g]$ in such a way that $C_{g}^{o}$ is the isometries of $g$. Therefore $C_{g}^{o}$ acts on the complex (2.1) so that this action induces the action on the cohomology groups.

We see from Theorems 2.1, 2.2 that $\hat{M}(X)$ has dimension at most 57 . Moreover, we have the following proposition from which $\hat{M}(X)$ is at [g] locally an analytic subset of $\boldsymbol{H}^{1} \cong \boldsymbol{R}^{57}$.

Proposition 2.3. Let $g$ be a Ricci flat metric on a K3 manifold $X$. Then the conformal group $C_{g}^{o}(X)=\left\{\mathrm{id}_{X}\right\}$. Moreover $C_{g}^{\prime}(X)=\left\{\mathrm{id}_{X}\right\}$ where $C_{g}(X)=$ $C_{g} \cap \operatorname{Diff}^{\prime}(X)$.

Proof. As shown in the proof of Proposition 2.1, $C_{g^{\prime}}$ consists of isometries of $g$. So, let $\phi$ be an isometry of $g$ in Diff'. Since $\phi^{*}$ acts as the identity on $H^{2}(X, \boldsymbol{Z})$ and hence on $H^{2}(X, \boldsymbol{R}), \phi^{*}=$ id on $H^{2}(g)$ and then $\phi$ must be an automorphism of $X$ with respect to a complex structure $J=J_{\alpha}$ induced from a certain self-dual harmonic 2-form $\alpha$. Therefore $\phi=\mathrm{id}_{X}$ by Proposition 11.3 (the weak Torelli theorem), § VIII in [4]. This completes the proof.

Proposition 2.4 (Proposition 4.2, [11]). The first cohomology group $\boldsymbol{H}^{1}$ at $[g]$, where $g$ is Ricc flat, is isomorphic to the tensor product $H^{-}(g) \otimes\left(H^{+}(g)\right)^{*}$.

Proof. It suffices to show the following.

Let $\phi_{a}^{+} \in H^{+}(g), a=1,2,3$ and $\phi_{b}^{-} \in H^{-}(g), b=1, \cdots, 19$ be harmonic self-dual (anti-self-dual) 2-forms which constitute orthonormal bases of $H^{+}(g)$ and $H^{-}(g)$, respectively. Then via the identification $\mathrm{H}^{+} \cong\left(\mathrm{H}^{+}\right) *$ the tensor products

$$
\psi_{b}^{-} \otimes \psi_{a}^{+} \in H^{-} \otimes H^{+}, \quad 1 \leqq a \leqq 3,1 \leqq b \leqq 19
$$

form an orthonormal basis of $\boldsymbol{H}^{1}$ with respect to the $L^{2}$-metric.

So, identify

$$
\begin{aligned}
& \Omega^{-} \otimes \Omega^{+}\left(\cong \operatorname{Hom}\left(\Omega^{+}, \Omega^{-}\right)\right) \stackrel{\cong}{\longrightarrow} \mathrm{S}_{o}^{2}\left(T^{*}\right) \\
& \left(\eta^{-}, \eta^{+}\right) \longmapsto h=\left(h_{\imath \jmath}\right)
\end{aligned}
$$

by $h_{\imath j}=\sum_{k l} \bar{g}^{k l} \eta_{\bar{i} k} \eta_{l j}^{+}$.

For $\psi^{+} \in H^{+}, \psi^{-} \in H^{-}$we let $h \in \Gamma\left(\mathrm{S}_{o}^{2}\left(T^{*}\right)\right)$ be given by $\psi^{-} \otimes \psi^{+}$via the above identification. To show $h \in \operatorname{Ker} L^{*} \cap \operatorname{Ker} D$ we first check that $L^{*}(h)=0$ and then $D(h)=0$.

We see easily $L^{*}(h)=0$ because $L^{*}(h)$ is given by $\left(L^{*}(h)\right)_{i}=-\sum_{j k} g^{j k} \nabla_{k} h_{j i}$. Since $g$ is Ricci flat, $D(h)$ is for any $h$ the $\mathrm{S}_{0}^{2}\left(\Omega^{+}\right)$-component of $U(h) \in$ 
$\Gamma\left(\Omega^{2} \otimes \Omega^{2}\right)$, defined by

$$
U(h)_{i j k l}=1 / 2\left(\nabla_{k} \nabla_{j} h_{i l}-\nabla_{l} \nabla_{j} h_{i k}-\nabla_{k} \nabla_{i} h_{j l}+\nabla_{l} \nabla_{i} h_{j k}\right)
$$

so that for our $h$

$$
U(h)_{i j k l}=1 / 2\left\{\left(\nabla_{k} \nabla_{s} \psi_{\overline{i j}}\right)\left(\psi^{+}\right)_{l}^{s}-\left(\nabla_{l} \nabla_{s} \psi_{\overline{i j}}\right)\left(\psi^{+}\right)_{k}^{s}\right\} .
$$

Now we may assume $\phi^{+}=\omega$ the Kähler form. So by using the complex indices we can show that $D(h)=0$.

It is not hard to show by Proposition 2.1 that $\psi_{a}^{-} \otimes \psi_{b}^{+}, 1 \leqq a \leqq 19,1 \leqq b \leqq 3$ gives a basis, since the $L^{2}$-inner product of $\phi_{a}^{-} \otimes \psi_{b}^{+}$and $\phi_{c}^{-} \otimes \psi_{d}^{+}$is $\delta_{a c} \delta_{b d}$.

Now we divide the moduli into three parts according to the sign of the Yamabe invariant, in other words, the sign of constant scalar curvature;

$$
\mathscr{M}(X)=\mathscr{M}^{(+)} \sqcup \mathscr{M}^{(0)} \sqcup \mathscr{M}^{(-)},
$$

where the Yamabe invariant is a conformal invariant of a conformal structure, which is essentially the value of the constant scalar curvature of a Yamabe metric (see [9]). A similar division is given for other moduli $\hat{\mathscr{M}}(X)$ and $\tilde{\mathscr{M}}(X)$.

Since a K3 manifold $X$ has $b^{+}>0$, the Weitzenböck-Bochner formula for harmonic self-dual 2-forms assures that there is no anti-self-dual conformal structure of positive Yamabe invariant. On the other hand, from Proposition 0.1 in Introduction any anti-self-dual conformal structure of zero Yamabe invariant has the unique Ricci flat metric of volume one as a specific representative. So we can identify $\mathscr{M}^{(0)}(X), \hat{\mathscr{M}}^{(0)}(X)$ and $\tilde{\mathscr{M}}^{(0)}(X)$ with $\mathcal{E}(X), \hat{\mathcal{E}}(X)$ and $\tilde{\mathcal{E}}(X)$, respectively.

Proposition 2.5. The mapping class group Diff'/Diffo acts freely on $\hat{\mathscr{M}}^{(0)}(X)$ and hence on $\hat{\mathcal{E}}(X)$.

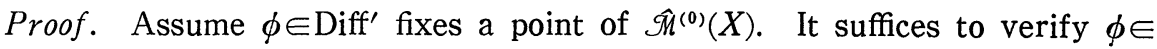
Diff $^{\circ}$. Let $[g]$ be a conformal structure representing this point. Then there is a $\phi_{1} \in$ Diff $^{\circ}$ such that $\phi^{*} g=\phi_{1}^{*} g$. So $\phi_{2}=\left(\phi_{\circ} \phi_{1}^{-1}\right) \in$ Diff $^{\prime}$ fixes $g$. Since $g$ is Ricci flat, it follows from the argument in the proof of Proposition 2.3 that $\phi_{2}=i d_{X}$, in other words, $\phi \in$ Diff $^{\circ}$. This proves Proposition 2.5.

We have the following diagram

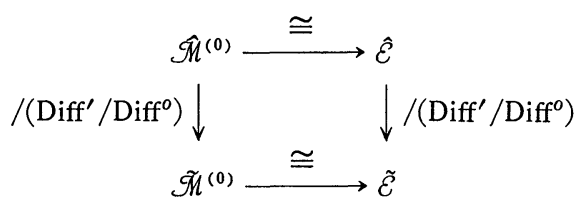

for which the Teichmüller moduli $\tilde{\mathcal{E}}$ is the quotient of $\hat{\mathcal{E}}$ by the fixed-point free action of the discrete group $\operatorname{Diff}^{\prime} / \operatorname{Diff}^{o}$. Since, as explained in $\S 1, \tilde{\mathcal{E}}$ has a 
smooth manifold structure, $\hat{\mathcal{E}}$ has also a smooth manifold structure. It is a covering space over a simply connected manifold $\tilde{\mathcal{E}}$ and is then considered as a union of copies of $\tilde{\mathcal{E}}$, where the number of copies is the order $\# \operatorname{Diff}^{\prime} /$ Diff $^{\circ}$.

Return back to the moduli of conformal structures $\hat{\mathscr{M}}^{(0)}(X)$ and $\tilde{\mathscr{M}}^{(0)}(X)$.

As was shown in Theorem 2.2 any point of $\hat{\mathscr{M}}^{(0)}(X)$ has a neighborhood in $\hat{M}(X)$ of the form $\left\{h \in \boldsymbol{H}^{1} ;|h|<\varepsilon, \Psi(h)=0\right\}$.

On the other hand, via the identification $\hat{\mathscr{M}}^{(0)}(X) \cong \hat{\mathcal{E}}(X)$ and by the argument just above the point in $\hat{\mathcal{E}}(X)$ corresponding to this point has a neighborhood in the Grassmannian manifold $G r_{3,22}^{+}$from Theorem 1.2. This neighborhood is considered as an $\varepsilon$-neighborhood in $H^{-}(g) \otimes\left(H^{+}(g)\right)^{*}$, which is from the Grassmannian space structure just the tangent space at the 3-plane $H^{+}(g)$.

Since $\boldsymbol{H}^{1} \cong H^{-}(g) \otimes\left(H^{+}(g)\right)^{*}$ by Proposition 2.4 and an $\varepsilon$-neighborhood in $\boldsymbol{H}^{1}$ corresponds to an $\varepsilon^{\prime}$-neighborhood of $H^{-}(g) \otimes\left(H^{+}(g)\right)^{*}$, we have the

ASSERTION. The map $\Psi ; \boldsymbol{H}^{1} \rightarrow \boldsymbol{H}^{2}$ must be trivial for any Ricci flat metric $g$.

Thus the following is verified.

THEOREM 2.6. The moduli $\hat{\mathscr{M}}^{(0)}(X)$ of anti-self-dual conformal structures of zero Yamabe invariant is an open subset of $\hat{M}(X)$ and has a smooth manifold structure (possibly not connected) of dimension 57, isomorphic to $\hat{\mathcal{E}}(X)$.

Remark. It is not known whether $\hat{\mathscr{M}}^{(-)}(X)$ is empty or not.

CONJECTURE. The mapping class group of second kind Diff'/Diffo consists only of the identity, or equivalently $\hat{\mathcal{E}}(X)$ corncides with $\tilde{\mathcal{E}}(X)$.

Remark that the mapping class group $\operatorname{Diff}^{+} /$Diff $^{\prime}$ of a $\mathrm{K} 3$ manifold $X$ is identified with the index two subgroup Aut $\left(H^{2}(X, Z), q_{X}\right) \cap S O(3,19)$ of the group Aut $\left(H^{2}(X, \boldsymbol{Z}), q_{X}\right)$ of automorphisms ([5]). There are certain criteria on the mapping class group relative to the lattice $\left(H^{2}(M, Z), q_{M}\right)$ derived from the surgery theory.

Actually, Wall showed the following ([19]); let $N$ be a simply connected, compact oriented 4-manifold with indefinite cup product $q_{N}$ or of rank $H^{2}(N) \leqq 8$. If $M$ is a connected sum $N \#\left(S^{2} \times S^{2}\right)$, then every positive $q_{M}$-automorphism of $H^{2}(M, Z)$ is induced by a $\phi \in \operatorname{Diff}^{+}(M)$.

On the other hand, M. Kreck obtained in [17] that if $M$ is a simply connected compact, connected 4-manifold, then the group consisting of $\phi \in \operatorname{Diff}^{+}(M)$ which is pseudo-isotopic to $i d_{M}$ is isomorphic to $\operatorname{Diff}^{\prime}(M)$. Here $\phi$ is pseudoisotopic to $\phi^{\prime}$ if there is a diffeomorphism $F$ of the product space $M \times[0,1]$ such that $F(x, 0)=(\phi(x), 0)$ and $F(x, 1)=\left(\phi^{\prime}(x), 1\right)$ (see [6]). 


\section{$\S 3$. $\quad L^{2}$-metric on the moduli of conformal structures}

In this section we will see how an $L^{2}$-metric is defined on the moduli of anti-self-dual conformal structures on a general 4-manifold $M$.

Since such a moduli is embedded in the ambient moduli, the moduli of all conformal structures, in order to get such an $L^{2}$-metric we will show that the space $\mathcal{C}(M)$ of all conformal structures on $M$ admits an $L^{2}$-metric $\mathcal{G}$ which is diffeomorphism-invariant, namely $\phi^{*}\left(\mathcal{G}_{[g]}\right)=\mathcal{G}_{\phi^{*[g]}},[g] \in \mathcal{C}(M), \phi \in \mathrm{Diff}^{+}$.

Let $[g] \in \mathcal{C}(M)$. The tangent space $T_{[g]}$ to $\mathcal{C}(M)$ at $[g]$ is then given by the space $\Gamma\left(M, \mathrm{~S}_{o}^{2}\left(T^{*}\right)\right)$ of tracefree symmetric covariant tensors $h=\left(h_{\imath j}\right)$. Moreover by the identification (2.3) $T_{[g]}$ is identified with $\Gamma\left(M, \operatorname{Hom}\left(\Omega_{g}^{+}, \Omega_{g}^{-}\right)\right.$). So one can define an inner product $\mathcal{G}$ by

$$
\mathcal{G}(A, B)=1 / 2 \int_{M}-\operatorname{tr}\left(A B^{*}+B A^{*}\right) d V_{g}
$$

for $A, B \in \Gamma\left(M\right.$, Hom $\left.\left(\Omega^{+}, \Omega^{-}\right)\right)$(see Theorem 5 in [11]).

Here $A^{*}, B^{*} \in \Gamma\left(M, \operatorname{Hom}\left(\Omega^{-}, \Omega^{+}\right)\right)$mean the adjoint of $A, B$ with respect to the wedge product, respectively, that is, $(A(\alpha)) \wedge \beta=\alpha \wedge\left(A^{*}(\beta)\right)$ for any $\alpha \in \Omega^{+}$ and $\beta \in \Omega^{-}$.

We need the minus sign in (3.1) since $\operatorname{tr}\left(A A^{*}\right)$ is negative definite. This negative definiteness stems from that the wedge product $\wedge$ is positive on $\Omega^{+}$ and is negative on $\Omega$.

The action of diffeomorphism on $\mathcal{C}(M)$ induces the differential map $T_{[g]} \rightarrow$ $T_{\left[\phi^{*} \mathrm{~g}\right]}, A \mapsto \phi^{*}(A)=\left(\left(\phi^{*}\right)^{-1} \circ A \circ \phi^{*}\right)$.

So one has $\operatorname{tr}\left(\left(\phi^{*} A\right)\left(\phi^{*} B\right)^{*}\right)(x)=\operatorname{tr}\left(A B^{*}\right)(\phi(x))=\left(\phi^{*} \operatorname{tr}\left(A B^{*}\right)\right)(x), x \in M$.

In order that the $G$ depends only on a conformal structure, not on a choice of representative metric $g$ we require that the volume form $d \boldsymbol{V}, g \mapsto d \boldsymbol{V}_{g}$, appeared in the definition (3.1), must satisfy the conformally invariant property $d \boldsymbol{V}_{f g}=d \boldsymbol{V}_{g}$. Moreover we require that $d \boldsymbol{V}_{\phi^{*} g}=\phi^{*}\left(d \boldsymbol{V}_{g}\right)$, the diffeomorphism naturality, because of the diffeomorphism-invariance of $\mathcal{G}$.

We call such a volume form a canonical volume form.

Proposition 3.1. If $M$ has $b^{+}(M)>0$ or $b^{-}(M)>0$, then $M$ admits a canonical volume form $d \boldsymbol{V}$.

Proof. Assume for brevity $b^{+}>0$. Let $[g] \in \mathcal{C}(M)$ and $g$ be a metric representing it. To prove the proposition we choose a basis $\left\{\phi_{i}\right\}$ of $H^{+}(M, g)$ which is $q_{M}$-orthonormal, that is, $q_{M}\left(\left[\phi_{i}\right],\left[\phi_{j}\right]\right)=\delta_{i j}$ as cohomology classes, or equivalently $\int_{M} \phi_{i} \wedge \phi_{j}=\delta_{i j}$.

We define then $d \boldsymbol{V}_{g}$ by

$$
d \boldsymbol{V}_{g}=\sum_{i=1}^{b+} \phi_{i} \wedge \phi_{i}
$$


Although this volume form $d \boldsymbol{V}_{g}$ may be degenerate, it is positive almost everywhere. This is because $\phi_{i} \wedge \phi_{i}=\left|\phi_{i}\right|^{2} d v_{g}$ and $d \boldsymbol{V}_{g}$ vanishes exactly at points where all $\phi_{i}$ 's vanish, and any nonzero harmonic 2 -form does not vanish almost everywhere.

To show the diffeomorphism naturality we first remark that any $\phi \in$ Diff $^{+}$ induces the $q_{M}$-isometry; $H^{+}(M, g) \rightarrow H^{+}\left(M, \phi^{*} g\right)$. So $\left\{\phi^{*} \phi_{i}\right\}$ is a $q_{M}$-orthonormal basis of $H^{+}\left(M, \phi^{*} g\right)$. Therefore,

$$
d \boldsymbol{V}_{\phi * g}=\sum_{i}\left(\phi^{*} \psi_{i} \wedge \phi^{*} \phi_{i}\right)=\phi^{*}\left(\sum_{i} \phi_{i} \wedge \phi_{i}\right)=\phi^{*}\left(d \boldsymbol{V}_{g}\right)
$$

If $b^{+}(M)=0$ but $b^{-}(M)>0$, then we need a minor change in the definition (3.2), only the minus sign.

One can check the diffeomorphism-invariance of the $L^{2}$-metric $\mathcal{G}$, since the integration over $M$ is preserved by the action of diffeomorphisms so that

$$
\int_{M} \operatorname{tr}\left\{\left(\phi^{*} A\right)\left(\boldsymbol{\phi}^{*} B\right)^{*}+\left(\boldsymbol{\phi}^{*} B\right)\left(\boldsymbol{\phi}^{*} A\right)^{*}\right\} d \boldsymbol{V}_{\phi^{*} g}=\int_{M} \boldsymbol{\phi}^{*}\left(\operatorname{tr}\left(A B^{*}+B A^{*}\right)\right) \phi^{*}\left(d \boldsymbol{V}_{g}\right)
$$

reduces to $\int_{M} \operatorname{tr}\left(A B^{*}+B A^{*}\right) d \boldsymbol{V}_{g}$.

The $L^{2}$-metric $\mathcal{G}$ on $\mathcal{C}(M)$ descends to the quotient spaces $\mathcal{C}(M) / \mathrm{Diff}^{+}$, $\mathcal{C}(M) /$ Diff $^{\prime}$ and $\mathcal{C}(M) /$ Diff $^{\circ}$, respectively so that

THEOREM 3.2. If $b^{+}(M)>0$ or $b^{-}(M)>0$, then the moduli admits an $L^{2}$-metric in such a way that (i) each of the following projections is isometric;

$$
\hat{\mathscr{N}}(M) \longrightarrow \tilde{M}(M) \longrightarrow \mathscr{M}(M)
$$

and (ii) the mapping class groups $\Gamma^{\prime}(M)=\operatorname{Diff}^{\prime}(M) / \operatorname{Diff}^{\circ}(M)$ and $\Gamma(M)=$ $\operatorname{Diff}^{+}(M) /$ Diff' $^{\prime}(M)$ act as isometries on $\hat{\mathscr{M}}(M)$ and $\tilde{\mathscr{M}}(M)$, respectively.

The following is then obtained.

THEOREM 3.3. The $L^{2}$-metric $\mathcal{G}$ on the moduli $\tilde{M}^{(0)}(X)$ of anti-self-dual conformal structures of zero Yamabe invariant on a K3 manifold $X$ is isometric up to constant to the invariant metric defined at (2.4) on the Teichmüller moduli $\tilde{\mathcal{E}}(X)$.

Proof. For any Ricci flat metric $g$ representing a conformal structure $[g]$ $H^{+}(g)$ consists only of parallel self-dual 2-forms so that each member of an orthonormal basis $\left\{\psi_{i}\right\}$ has the same constant norm. Thus the canonical volume form is just a constant multiple of the ordinary Riemannian volume form $d v_{g}$ of $g$.

By Proposition 2.4 and Theorem 2.6 the basis $\left\{\phi_{i}\right\}$ together with an orthonormal basis $\left\{\psi_{j}^{-}\right\}$of $H^{-}(g)$ give via the identification $\boldsymbol{H}^{1} \cong H^{-}(g) \otimes H^{+}(g)$ an orthonormal basis $\left\{\psi_{j}^{-} \otimes \psi_{\imath}^{+}\right\}$of the tangent space $T_{[g]}$ of the moduli $\tilde{\mathscr{M}}^{(0)}$ with 
respect to the $L^{2}$-metric $\mathcal{G}$ and gives again by the period map theorem (Theorem 1.2) together with the formula (1.5) an orthonormal basis, up to constant, of the tangent space of the Grassmannian manifold $G r_{3,22}^{+}$at the corresponding point.

Remark 1. Since the invariant metric is complete on $\mathrm{Gr}_{3,22}^{+}$, but not complete on $T$, the $L^{2}$-metric $\mathcal{G}$ is non-complete on $\widetilde{M}^{(0)}(X)$. The metric completion of $\tilde{M}^{\left({ }^{0}\right)}(X)$ or of $\tilde{\mathcal{E}}(X)$ relative to the $L^{2}$-metric is the space which is isometrically identified with the symmetric space $G r_{3,22}^{+}$.

Remark 2. The complement of $T, G r_{3,22}^{+} \backslash T$ consists of orbifold-singular Ricci flat metrics on $X$ ([1], [13]). Here an orbifold-singular Ricci flat metric on $X$ is a $C^{\infty}$ symmetric covariant 2-tensor on $X$ of the form $\pi^{*} g$, where $\pi: M \rightarrow V$ is a surjective real analytic map to a Ricci flat Einstein orbifold $(V, g)$.

Moreover in the completion procedure we observe the bubbling off phenomena ([13], [3]). In fact from Theorem 21 in [13], if $\left\{g_{i}\right\}$ is a sequence in $T$ having the limit $g \in G r_{3,22}^{+} \backslash T$, then (i) the curvature of $g_{2}$ concentrates near some configurations $E$ of embedded 2-spheres of self-intersection number -2 , (ii) if we rescale $g_{\imath}$ by the local maximum value of the curvature, then the rescaled metrics $\tilde{g}_{\imath}$ converge to ALE gravitational instantons corresponding to the simple singularities obtained by contracting the configurations $E$ and (iii) outside the singularities $g_{\imath}$ converges to the orbifold-singular metric $g$.

\section{$\S 4$. The moduli on an Enriques manifold}

Let $Y$ be an Enriques manifold, namely a compact 4-manifold underlying an Enriques surface. Here an Enriques surface is a compact complex surface obtained by a holomorphic $\boldsymbol{Z}_{2}$-quotient of a $\mathrm{K} 3$ surface. Then $Y$ is a $\boldsymbol{Z}_{2}$-quotient of a K3 manifold $X$. Note that an Enriques surface has the trivial bundle $K^{\otimes 2}$ ([15, § VIII in 4]).

Let $\pi: X \rightarrow Y$ be a covering map yielding the Enriques manifold $Y$ and let $\sigma: X \rightarrow X$ be the deck transformation of $X, \pi \circ \sigma=\pi$ such that $Y=X /\langle\sigma\rangle$.

The topological invariants of $Y$ are $\chi(Y)=(1 / 2) \chi(X)=12, \tau(Y)=(1 / 2) \tau(X)=$ -8 and hence $b_{2}(Y)=10, b^{+}(Y)=1, b^{-}(Y)=9$.

The deck transformation $\sigma$ induces the cup product isometry of the cohomology group $L \equiv H^{2}(X, \boldsymbol{Z}) \cong \bigoplus^{2}\left(-E_{8}\right) \oplus \bigoplus^{3} H$ given by

$$
x \oplus y \oplus z_{1} \oplus z_{2} \oplus z_{3} \longmapsto y \oplus x \oplus-z_{1} \oplus z_{3} \oplus z_{2}
$$

so that the $\sigma$-invariant sublattice $L^{+}$is isomorphic to $-2 E_{8} \oplus 2 H$ and the unimodular lattice $1 / 2 L^{+}$, isomorphic to $-E_{8} \oplus H$, gives the cohomology group of an Enriques manifold $Y$ with the cup product $q_{Y}$ (see Lemma 19.1, § VIII in [4]) and thus $b^{+}=1, b^{-}=9$.

From Proposition 0.1 same as in the $\mathrm{K} 3$ manifold case we identify the moduli of unit volume Ricci flat metrics on $Y$ with the moduli of anti-self-dual 
conformal structures of zero Yamabe invariant.

Let $g$ be a Ricci flat metric on $Y$. Then, since $g$ is anti-self-dual, as discussed in $\S 2$, it provides an elliptic complex defining local deformation of antiself-dual conformal structures:

$$
0 \longrightarrow \Gamma(Y, T) \stackrel{L_{g}}{\longrightarrow} \Gamma\left(Y, \mathrm{~S}_{0}^{2}\left(T^{*}\right)\right) \stackrel{D_{g}}{\longrightarrow} \Gamma\left(Y, \mathrm{~S}_{o}^{2}\left(\Omega^{+}\right)\right) \longrightarrow 0 .
$$

The index is $h^{0}-h^{1}+h^{2}=-26$ from the topological invariants of $Y$.

Proposition 4.1. The elliptic complex (4.2) has the cohomology groups $\boldsymbol{H}^{0}=0$, $\boldsymbol{H}^{1} \cong \boldsymbol{R}^{29}$ and $\boldsymbol{H}^{2} \cong \boldsymbol{R}^{3}$ for an arbitrary Ricci flat metric.

Proof. We apply the same proof of Proposition 2.1 to show $\boldsymbol{H}^{0}=0$. We postpone the calculation of $h^{2}=\operatorname{dim} \boldsymbol{H}^{2}$ until just after Proposition 4.2. Actually we will see there $h^{2}=3$ and hence $h^{1}=29$.

The situation for an Enriques manifold is quite similar to the K3 manifold case. So, $\hat{\mathscr{M}}(Y)$ can be identified around the conformal structure [g] with the $C_{g^{o}}$-quotient of a real analytic variety $\left\{h \in \boldsymbol{H}^{1} ;|h|<\varepsilon, \Psi(h)=0\right\}$ where $\Psi: \boldsymbol{H}^{1} \rightarrow$ $\boldsymbol{H}^{2}$ is an analytic map, and $C_{g}^{o}=C_{g} \cap \operatorname{Diff}^{\circ}(Y)$ denotes the group of conformal transformations of $[g]$.

ASSERTION. $C_{g}^{o}=\left\{\operatorname{id}_{Y}\right\}$.

This is given as follows. Since $g$ is Ricci flat, $g$ is a Yamabe metric of zero Yamabe invariant so that $C_{g}^{o}$ consists only of isometries. Let $\phi$ be such an isometry. Then it lifts up as an isometry $\bar{\phi}$ of $\bar{g}$ commuting with $\sigma$ where $\bar{g}=\pi^{*} g$. Since $\phi \in \operatorname{Diff}^{\circ}, \bar{\phi}$ is also in Diff ${ }^{o}$ of $X$. It follows then from Proposition 2.3 that $\bar{\phi}$ is $i d_{X}$ and hence $\phi$ is $i d_{Y}$.

On the other hand, a Ricci flat metric $g$ on $Y$ lifts up to a Ricci flat metric $\bar{g}$ on $X$ which is deck transformation invariant and vice versa so that one has

OBSERVATION. The moduli of Ricci flat metrics on $Y$ is considered as the space of $\sigma$-invariant Ricci flat metrics $\bar{g}$ on $X$ of $\operatorname{Vol}(\bar{g})=2$.

Suppose that $\bar{g}$ is a Ricci flat metric on a K3 manifold $X$ such that $\bar{g}=\pi^{*} g$. Then we have the elliptic complex (2.1) over $X$ associated to $\bar{g}$. Because $\bar{g}$ is $\sigma$-invariant, i. e., $\sigma^{*} \bar{g}=\bar{g}$, the deck transformation $\sigma$ induces the involutive endomorphism of the elliptic complex (2.1).

If we restrict ourself to the $\sigma$-fixed parts, we derive the $\sigma$-invariant elliptic complex

$$
0 \longrightarrow \Gamma_{\sigma}(X, T) \longrightarrow \Gamma_{\sigma}\left(X, \mathrm{~S}_{o}^{2}\left(T^{*}\right)\right) \longrightarrow \Gamma_{\sigma}\left(X, \mathrm{~S}_{o}^{2}\left(\Omega^{+}\right)\right) \longrightarrow 0
$$

with cohomology groups $\boldsymbol{H}_{\sigma}^{i}, i=0,1,2$. 
Proposition 4.2. Each cohomology group $\boldsymbol{H}_{\sigma}^{i}$ is canonically $\imath$ dentified with the linear subspace of $\boldsymbol{H}^{i}(X)$ of $X$ consisting of $\sigma$-fixed vectors. $\boldsymbol{H}^{i}(X)$.

So, we identify $\boldsymbol{H}_{\sigma}^{i}$ with the elementwise $\sigma$-fixed linear subspace, $\boldsymbol{H}_{\sigma}^{i} \subset$

Proof. For brevity we show the case $i=1$. By definition each $\bar{h}$ of $\boldsymbol{H}_{\sigma}^{1}$ satisfies $D_{\bar{s}} \bar{h}=0$ and $\left(L_{\bar{g}} \bar{U}, \bar{h}\right)=0$ for all $\bar{U} \in \Gamma_{\sigma}(X, T)$.

Denote by $\left(\boldsymbol{H}^{1}\right)^{\sigma}$ the linear subspace in $\boldsymbol{H}^{1}(X)$ of the above proposition. Then we observe $\left(\boldsymbol{H}^{1}\right)^{\sigma} \subset \boldsymbol{H}_{\sigma}^{1}$. Now we prove the converse implication. Let $\bar{h} \in \boldsymbol{H}_{\sigma}^{1}$. Consider this as a section of $\mathrm{S}_{0}^{2}\left(T^{*}\right)$. So from the harmonic decomposition $\bar{h}=\bar{h}_{1}+\bar{h}_{2}+\bar{h}_{3}, \bar{h}_{1} \in \boldsymbol{H}^{1}(X), \bar{h}_{2} \in \operatorname{Im} L_{\bar{g}}, \bar{h}_{3} \in \operatorname{Im} D_{\bar{g}}^{*}$. Since $D_{\bar{g}} \bar{h}=0$, it follows that $\bar{h}_{3}=0$. Then by the $\sigma$-invariance of $\bar{h}$ we can write $\bar{h}_{2}=L_{\bar{g}} \bar{U}, \bar{U} \in \Gamma_{\sigma}(T)$. Substitute this equality into $\left(L_{\bar{g}} \bar{V}, \bar{h}\right)=0$ to get $L_{\bar{g}} \bar{U}=0$. So the proof is completed.

We are now ready to complete the proof of Proposition 4.1, namely to show $\operatorname{dim} \boldsymbol{H}^{2}=3$ for each Ricci flat metric on an Enriques manifold $Y$.

By Proposition 4.2 it is sufficient to assert $\operatorname{dim} \boldsymbol{H}_{\sigma}^{2}=3$ for any Ricci flat metric $\bar{g}$ such that $\sigma^{*} \bar{g}=\bar{g}$. As we showed in the proof of Proposition 2.1, $\boldsymbol{H}^{2}$ consists of parallel sections. Those sections of $\mathrm{S}_{0}^{2}\left(\Omega^{+}\right)$are of the form $\Sigma_{a, b} \psi_{a}^{+} \otimes \psi_{b}^{+}$, where $\psi_{a}^{+}, a=1,2,3$ are parallel self-dual 2 -forms giving a basis of $H^{+}(\bar{g})$.

Before counting the dimension we prepare the following

Proposition 4.3. For a o-invariant Ricci flat metric $\bar{g}$ on $X H^{+}(\bar{g})$ and $H^{-}(\bar{g})$ split as

$$
\begin{aligned}
& H^{+}(\bar{g})=W_{1}^{+} \oplus W_{2}^{+}, \\
& H^{-}(\bar{g})=W_{1}^{-} \oplus W_{2}^{-},
\end{aligned}
$$

into the subspaces of dimension $\operatorname{dim} W_{1}^{+}=1, \operatorname{dim} W_{2}^{+}=2, \operatorname{dim} W_{1}^{-}=9$ and $\operatorname{dim} W_{2}^{-}=10$ such that $\sigma^{*}=i d$ on $W_{1}^{ \pm}$and $\sigma^{*}=-i d$ on $W_{2}^{ \pm}$.

Proof. This proposition is obvious, since the deck transformation $\sigma$ acts on $H^{2}(X, Z)$ as $(4.1)$, or equivalently $b^{+}=1$ and $b^{-}=9$ for an Enriques manifold.

Return back to the counting. From this proposition the action of $\sigma$ on $H^{+}(\bar{g})$ is

$$
\sigma^{*} \psi_{1}^{+}=\phi_{1}^{+}, \quad \sigma^{*} \psi_{a}^{+}=-\phi_{a}^{+}, \quad a=2,3
$$

for a certain basis $\left\{\phi_{a}^{+}\right\}$so we see easily that $\phi_{1}^{+} \otimes \phi_{1}^{+}-\phi_{2}^{+} \otimes \psi_{2}^{+}, \phi_{1}^{+} \otimes \psi_{1}^{+}-\phi_{3}^{+} \otimes \phi_{3}^{+}$ and $\psi_{2}^{+} \otimes \psi_{3}^{+}+\psi_{3}^{+} \otimes \psi_{2}^{+}$give a basis of the $\sigma$-invariant linear subspace $\boldsymbol{H}_{\sigma}^{2}$.

The complex (4.3) is just the involution-invariant version of (4.2) so that $\hat{M}^{(0)}(Y)$ and $\hat{\mathcal{E}}(Y)$ for an Enriques manifold $Y$ are investigated by means of involution-invariant portions of the corresponding $\hat{\mathscr{M}}^{(0)}(X)$ and $\hat{\mathcal{E}}(X)$ for a $\mathrm{K} 3$ 
manifold $X$.

Let $[g]$ be an anti-self-dual conformal structure on an Enriques manifold $Y$. Assume that it has zero Yamabe invariant. Then it is represented by a Ricci flat metric $g$.

From the real analytic variety theorem a neighborhood of $[g]$ in the moduli $\hat{\mathscr{M}}(Y)$ has the form of the zero locus of $\Psi_{\sigma}$ in an $\varepsilon$ ball $\subset \boldsymbol{H}_{\sigma}^{1}$, where $\Psi_{\sigma}: \boldsymbol{H}_{\sigma}^{1} \rightarrow \boldsymbol{H}_{\sigma}^{2}$ is the analytic map associated to the $\sigma$-invariant Kuranishi map. By Proposition 4.2 we can consider $\Psi_{\sigma}$ just as $\Psi: \boldsymbol{H}^{1} \rightarrow \boldsymbol{H}^{2}$ over $X$ restricted to the $\sigma$-fixed linear subspace. As was proved in $\S 2$, we have $\Psi=0$ which assures that at $[g] \hat{\mathscr{M}}(Y)$ is isomorphic to $\left\{\bar{h} \in \boldsymbol{H}^{1}(X) ;|\bar{h}|<\varepsilon, \boldsymbol{\sigma}^{*} \bar{h}=\bar{h}\right\}$.

Because an $\varepsilon$-neighborhood of the first cohomology group over $X$ gives a neighborhood of $\hat{\mathscr{M}}^{(0)}(X)$, we can get a neighborhood of $[g]$ in $\hat{M}(Y)$ exactly inside the proper submoduli $\hat{\mathscr{M}}^{(0)}(Y)$, the moduli of anti-self-dual conformal structures of zero Yamabe invariant. Thus we get

THEOREM 4.4. The moduli $\hat{M}^{(0)}(Y)$, isomorphic to $\hat{\mathcal{E}}(Y)$, is a smooth manifold of dimension 29, whose tangent space is modelled by $\boldsymbol{H}_{\sigma}^{1}$, the elementwise $\sigma$ fixed linear subspace $\boldsymbol{H}^{\mathbf{1}}(X)$.

Since any Ricci flat metric $g$ on $Y$ induces a metric $\bar{g}$ on $X$ which is Ricci flat, we have a natural map

$$
\begin{aligned}
& \iota: \hat{\mathcal{E}}(Y) \longrightarrow \hat{\mathcal{E}}(X) \\
& g \bmod \operatorname{Diff}^{o}(Y) \longmapsto \bar{g} \bmod \operatorname{Diff}^{\circ}(X)
\end{aligned}
$$

In fact, arbitrary $\phi \in \operatorname{Diff}^{\circ}(Y)$ induces uniquely $\bar{\phi} \in \operatorname{Diff}^{\circ}(X)$, because $\phi$ is generated by finite number of vector fields on $Y$ and these vector fields lift up on $X$.

THEOREM 4.5. The map $\iota: \hat{\mathcal{E}}(Y) \rightarrow \hat{\mathcal{E}}(X)$ gives an embedding and moreover the image of this map is a totally geodesic submanifold of $\hat{\mathcal{E}}(X)$ equipped with the $L^{2}$-metric.

Proof. Let $g$ and $g_{1}$ be Ricci flat metrics on $Y$ such that the lifted Ricci flat metrics $\bar{g}$ and $\bar{g}_{1}$ satisfy $\bar{g}_{1}=\bar{\phi}^{*} \bar{g}$ for a $\bar{\phi} \in \operatorname{Diff}^{\circ}(X)$. Since $\bar{g}$ and $\bar{g}_{1}$ are $\sigma$ invariant, it holds $\sigma^{*} \bar{\phi}^{*} \bar{g}=\bar{\phi}^{*} \sigma^{*} \bar{g}$.

Hence $\bar{\phi} \circ \sigma \circ(\bar{\phi})^{-1} \circ \sigma \in C_{\bar{g}} \cap \operatorname{Diff}^{\prime}(X)$. Because by Proposition 2.3 $C_{\bar{g}} \cap \operatorname{Diff}^{\prime}(X)$ consists only of $i d_{X}, \bar{\phi}$ commutes with the deck transformation $\sigma$ so that $\bar{\phi}$ descends to a $\phi \in \operatorname{Diff}^{\circ}(Y)$ such that $g_{1}=\phi^{*} g$. So the map c is injective.

From the identification $\hat{\varepsilon} \cong \hat{M}^{(0)}$ for both $Y$ and $X$ and the first cohomology groups give their local coordinates, it is seen that $c$ is smooth and has at every point the maximal rank $\operatorname{dim} \boldsymbol{H}_{\sigma}^{1}$. Thus $\iota$ is an embedding.

To show the image is totally geodesic it suffices to verify that the image is exactly the fixed points of an isometry in $\hat{\varepsilon}(X)$. This isometry is just the action of the deck transformation $\sigma ; \bar{g} \bmod \operatorname{Diff}^{\circ}(X) \mapsto \sigma^{*} \bar{g} \bmod \operatorname{Diff}^{\circ}(X)$. We must check that the action of $\sigma$ is isometric. But we observed already at 
Theorem 3.2 that any orientation-preserving diffeomorphism preserves the conformally defined $L^{2}$-metric $\mathcal{G}$ on $\hat{\mathscr{M}}^{(0)}$ and this metric agrees with the invariant metric on $\hat{\mathcal{E}}(X)$ via the period map (see Theorem 3.3 and also Theorem $6,[10]$ ). Thus $\sigma$ yields an isometric transformation of $\hat{\mathscr{M}}^{(0)}(X) \cong \hat{\mathcal{E}}(X)$ preserving this identification.

It is not hard to see that the image of $c$ is exactly the fixed points of this isometry.

The isometry $\sigma: \hat{\mathcal{E}}(X) \rightarrow \hat{\mathcal{E}}(X)$ gives rise to an involutive isometry of the Teichmüller moduli, denoted by $\sigma: \tilde{\mathcal{E}}(X) \rightarrow \tilde{\mathcal{E}}(X)$, because $\sigma \circ \phi^{\circ} \sigma^{-1} \in$ Diff $^{\prime}$ for any $\phi \in$ Diff $^{\prime}$ in such a way that the following diagram commutes

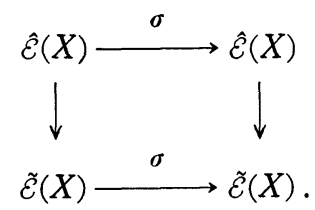

To obtain geometrical feature of $\tilde{\varepsilon}(Y)$ for an Enriques manifold $Y$ we investigate the space of $\sigma$-fixed points in $\tilde{\mathcal{E}}(X)$ which we denote by $\tilde{\mathcal{E}}_{\sigma}(X)$.

So, we consider the period map $p e: \tilde{\mathcal{E}}(X) \rightarrow S O(3,19) / S O(3) \times S O(19)$. Then $\sigma$ acts naturally as an isometry on the symmetric space by sending any oriented positive 3-plane $\Pi$ to $\sigma * \Pi$ so that the actions of $\sigma$ commute through the period map. Therefore, the image $p e\left(\tilde{\mathcal{E}}_{\sigma}(X)\right)$ is an open dense subset of $(S O(3,19) /$ $S O(3) \times S O(19))_{\sigma}$, where $(S O(3,19) / S O(3) \times S O(19))_{\sigma}$ is the fixedpoint set of the isometry $\sigma$.

Obviously this fixedpoint set is a symmetric space of noncompact type, totally geodesically embedded in the ambient symmetric space.

Proposition 4.6 (Theorem 0.4 in Introduction). The fixedpoint set has the structure of quotient space of the following form

$$
\begin{aligned}
& (S O(3,19) / S O(3) \times S O(19))_{\sigma} \\
& \quad=(S O(1,9) / S O(1) \times S O(9)) \times(S O(2,10) / S O(2) \times S O(10)) .
\end{aligned}
$$

The latter space is well embedded in $S O(3,19) / S O(3) \times S O(19)$.

Proof. Let us assume that $\Pi$ is an arbitrary oriented positive 3-plane such that $\sigma^{*} \Pi=\Pi$. The $q_{X}$-orthogonal complement $\Pi^{\perp}$ is then $\sigma$-invariant. So we have splittings of these subspaces $\Pi, \Pi^{\perp}$ like Proposition 4.3. Therefore it is not difficult to get the proposition. 


\section{§5. A $Z_{2}$-quotient of Enriques manifold}

Consider in this section the moduli of Ricci flat metrics on a 4-manifold $Z$ last appeared in Theorem 0.2 , Introduction, namely a $Z_{2}$-quotient $Z$ of an Enriques manifold $Y$.

The 4-manifold $Z$ is written as $Z=Y /\langle\theta\rangle$ where $\theta \in \operatorname{Diff}^{+}(Y)$ is fixedpoint free and involutive. So, the Euler number $\chi(Z)$ is $\chi(Z)=(1 / 2) \chi(Y)=6$ and the index $\tau(Z)=(1 / 2) \tau(Y)=-4$. Since $\pi_{1}(Z)=\boldsymbol{Z}_{2} \times \boldsymbol{Z}_{2}$ and hence $b_{1}(Z)=0$, we have $b^{+}(Z)=0$ and $b^{-}(Z)=4$ so that the cup product $q_{Z}$ of $Z$ is negative definite.

Let $g$ be a Ricci flat metric on $Z$. Then it lifts up to a $\theta$-invariant Ricci flat metric $\bar{g}$ on $Y$. Since $\theta^{*} \bar{g}=\bar{g}, \theta$ induces an involutive action of $H^{+}(Y, \bar{g})$.

From $b^{+}(Y)=1$ and $b^{+}(Z)=0, \theta$ acts as $-i d$ on $H^{+}(Y, \bar{g})$, i. e., $\theta^{*}(\alpha)=-\alpha$ for $\alpha \in H^{+}(Y, \bar{g})$.

Since from Proposition $0.1 \bar{g}$ is an anti-self-dual metric of zero scalar curvature, each element of $H^{+}(Y, \bar{g})$ is parallel in such a way that a certain $\alpha \in$ $H^{+}(Y, \bar{g})$ gives the Kähler form to the metric $\bar{g}$ with respect to a certain complex structure $J ; \alpha(u, v)=\bar{g}(J(u), v)$. In other words, $\bar{g}$ is a Kähler metric on a complex surface $(Y, J)$. It follows then from $\theta^{*} \alpha=-\alpha, \theta^{*} \bar{g}=\bar{g}$ that $\theta^{*} J=$ $-J$, i. e., $\theta$ is an anti-holomorphic involution.

On the other hand, from the topological invariants $b^{-}(Y)=9, b^{-}(Z)=4$, the space $H^{-}(Y, \bar{g})$ splits as

$$
H^{-}(Y, \bar{g})=V_{1}^{-} \oplus V_{2}^{-} \quad \operatorname{dim} V_{1}^{-}=4, \quad \operatorname{dim} V_{2}^{-}=5
$$

where $\theta^{*}$ is $i d$ on $V_{1}^{-}$and $-i d$ on $V_{2}^{-}$.

In the same way as in the argument for an Enriques surface we have for $g$ an elliptic complex (2.1) whose index is $26 / 2=13$.

Proposition 5.1. The cohomology groups for a Ricci flat metric $g$ on $Z$ are $\boldsymbol{H}^{0}=0, \boldsymbol{H}^{1} \cong \boldsymbol{R}^{15}$ and $\boldsymbol{H}^{2} \cong \boldsymbol{R}^{2}$.

Proof. We see $\boldsymbol{H}^{0}=0$ in a same way as in the proof of Proposition 2.1.

Now we compute the dimension $\operatorname{dim} \boldsymbol{H}^{2}$. For this we apply Proposition 4.2 to our situation. Actually $\boldsymbol{H}^{2}$ for $Z$ is isomorphic to the $\theta$-invariant linear subspace $\left(\boldsymbol{H}^{2}\right)_{\theta}$ of $\boldsymbol{H}^{2}$ for a Ricci flat Enriques manifold $(Y, \bar{g})$. We can then follow the argument given in [12]. We note that $\bar{g}$ is a Kähler metric on an Enriques surface $(Y, J)$. The following decomposition of $\mathrm{S}_{0}^{2}\left(\Omega^{+}\right)$is valid for any complex Kähler surface $M([7],[10])$. As a real vector bundle

$$
\mathrm{S}_{o}^{2}\left(\Omega^{+}\right)=\boldsymbol{R} \Phi \oplus\left(K_{M}\right)_{\boldsymbol{R}} \oplus\left(K_{M}^{\otimes 2}\right)_{\boldsymbol{R}}
$$

where $\Phi$ is a certain parallel section of $\mathrm{S}_{o}^{2}\left(\Omega^{+}\right)$, and $K_{M}$ and $K_{M}^{\oplus 2}$ are the canonical line bundle of $M$ and its square, respectively. Moreover $\left(K_{M}\right)_{R}$ means the rank two real vector bundle induced from $K_{M}$.

Since $\boldsymbol{H}^{2}=\operatorname{Ker} D D^{*}$ and $D D^{*}=(\nabla \nabla)^{2}$ for an arbitrary Ricci flat 4-manifold, 
the subspace $\left(\boldsymbol{H}^{2}\right)_{\theta}$ consists of $\theta$-invariant parallel sections of the tracefree symmetric product bundle $\mathrm{S}_{0}^{2}\left(\Omega_{Y}^{+}\right)$. We have seen in the above that $\theta$ is antiholomorphic and $\theta^{*} \alpha=-\alpha$ where $\alpha$ is the Kähler form of the Kähler metric $\bar{g}$ $\left(\alpha \in H^{+}(Y, \bar{g})\right)$.

Moreover $\theta^{*} \beta=c \bar{\beta}, c \in C$ for a certain section $\beta$ of unit norm of $K_{Y}$. Because $\theta$ is involutive, $|c|$ must be equal to 1 .

Since $\Phi$ has the following form ([10])

$$
\Phi=\frac{1}{4} \alpha^{2}-\frac{1}{2} \beta \cdot \bar{\beta},
$$

$\Phi$ is $\theta$-invariant.

On the other hand, for an Enriques surface $Y K_{Y}$ is not trivial but $K_{Y}^{\otimes^{2}}$ is trivial as holomorphic bundles. So $K_{Y}$ does not admit but $K_{Y}^{\otimes 2}$ admits a global holomorphic and hence parallel section. This section may be identified with $\beta^{2}$. Thus we have two parallel sections of $\left(K_{Y}^{\otimes 2}\right)_{R}$ the real part $\Phi_{1}=1 / 2\left(\beta^{2}+\bar{\beta}^{2}\right)$ and the imaginary part $\Phi_{2}=1 / 2 \sqrt{-1}\left(\beta^{2}-\bar{\beta}^{2}\right)$.

Of course $\theta^{*} \Phi_{1}, \theta^{*} \Phi_{2}$ are parallel in $\Gamma\left(Y,\left(K_{Y}^{\otimes 2}\right)_{R}\right)$. Since $\theta^{*} \beta=c \bar{\beta}$ with $c$ of $|c|=1$, the $2 \times 2$ coefficient matrix of $\theta^{*} \Phi_{\imath}$ relative to $\Phi_{\imath}$ has trace zero and determinant -1 so that this matrix has eigenvalues $+1,-1$. Therefore, $\Gamma\left(Y,\left(K_{Y}^{\otimes^{2}}\right)_{\boldsymbol{R}}\right)$ has a 1-dim linear subspace generated by a +1 -eigensection. Thus we see $\operatorname{dim} \boldsymbol{H}^{2}=\operatorname{dim}\left(\boldsymbol{H}^{2}(Y)\right)_{\theta}=2$.

$\boldsymbol{H}^{1}$ has the dimension $\operatorname{dim} \boldsymbol{H}^{\mathbf{1}}=-($ index $)+\operatorname{dim} \boldsymbol{H}^{\mathbf{0}}+\operatorname{dim} \boldsymbol{H}^{2}=15$.

Therefore, in a quite similar way to the argument given for Theorem 4.2 we get

THEOREM 5.2. The isotopy-Teichmüller moduli $\hat{\mathscr{M}}^{(0)}(Z)$ of anti-self-dual conformal structures of zero Yamabe invariant, which is isomorphic to the isotopyTeichmüller moduli $\hat{\mathcal{E}}(Z)$ of Ricci flat metrics on $Z$, admits a $15 \mathrm{dim}$ smooth manifold structure whose tangent space at each point is modelled by $\boldsymbol{H}_{\theta}^{1}(Y)$, the elementwise $\theta$-fixed linear subspace of the first cohomology group $\boldsymbol{H}^{1}(Y)$ for an Enriques manifold $Y$.

Since $\pi_{1}(Z)=\boldsymbol{Z}_{2} \times \boldsymbol{Z}_{2}$, we can further regard $Z$ as a $\boldsymbol{Z}_{2} \times \boldsymbol{Z}_{2}$-quotient of a K3 manifold $X, Z=X /\langle\sigma, \theta\rangle$ where $\sigma, \theta$ are involutive diffeomorphisms of $X$ satisfying $\sigma \circ \theta=\theta \circ \sigma$.

So, via the covering map; $X \rightarrow Z$ an arbitrary Ricci flat metric $g$ on $Z$ is considered as a Ricci flat metric $\bar{g}$ on $X$ which is $\langle\sigma, \theta\rangle$-invariant, i. e., $\sigma^{*} \bar{g}=$ $\theta^{*} \bar{g}=\bar{g}$ and we have a natural map like the Ricci flat Enriques manifold case

$$
\begin{aligned}
& j: \hat{\mathcal{E}}(Z) \longrightarrow \hat{\mathcal{E}}(X) \\
& g \bmod \operatorname{Diff}^{\circ}(Z) \longmapsto \bar{g} \bmod \operatorname{Diff}^{\circ}(X)
\end{aligned}
$$

Theorem 5.3. The map $j: \hat{\mathcal{E}}(Z) \rightarrow \hat{\mathcal{E}}(X)$ enjoys a totally geodesic embedding 
and the image $\jmath(\hat{\mathcal{E}}(Z))$ is a fixedpoint set of the isometries induced from the deck transformations $\langle\sigma, \theta\rangle$ in $\hat{\mathcal{E}}(X)$.

The isometries $\sigma, \theta: \hat{\mathcal{E}}(X) \rightarrow \hat{\mathcal{E}}(X)$ yield isometries of the Teichmüller moduli $\tilde{\mathcal{E}}(X)$ and also of the Grassmannian manifold $S O(3,19) / S O(3) \times S O(19)$ such that the actions of these isometries commute via the period map pe: $\tilde{\mathcal{E}}(X) \rightarrow S O(3,19)$ $/ S O(3) \times S O(19)$. Thus we obtain Theorem 0.5 in the introduction.

\section{REFERENCES}

[1] M. T. Anderson, Moduli spaces of Einstein metrics on 4-manifolds, Bull. Amer. Math. Soc., 2 (1989), 275-279.

[2] M.F. Atiyah, N.J. Hitchin And I. M. Singer, Self-duality in four dimensional Riemannian geometry, Proc. Roy. Soc. Lond. Ser. A, 362 (1978), 425-461.

[3] S. Bando, A. Kasue AND H. NAKajima, On a construction of coordinates at infinity on manifolds with fast curvature decay and maximal volume growth, Invent. Math., 97 (1989), 313-349.

[4] W. Barth, C. Peters and A. Van de Ven, Compact Complex Surfaces, SpringerVerlag, Berlin-Heidelberg-New York-Tokyo, 1984.

[5] A.L. Besse, Einstein Manifolds, Springer-Verlag, Berlin-Heidelberg-New YorkLondon-Paris-Tokyo, 1987.

[6] J. CERF, The pseudo-isotopy theorem for simply connected differentiable manifolds, Manifolds-Amsterdam, Lecture Notes in Math., 197, Springer-Verlag, Berlin-Heidelberg-New York, 1970, 76-82.

[7] A. Derdzinski, Self-dual Kähler manifolds and Einstein manifolds of dimension four, Compositio Math., 49 (1983), 405-437.

[8] N. J. Hitchin, Compact four-dimensional Einstein manifolds, J. Differential Geom., 9 (1974), 435-441.

[9] M. Iтон, Yamabe metrics and the space of conformal structures, Internat. J. Math., 2 (1991), 659-671.

[10] M. Iтон, Half conformally flat structures and the deformation obstruction space, Tsukuba J. Math., 17 (1993), 143-158.

[11] M. Iтон, Moduli of half conformally flat structures, Math. Ann., 296 (1993), 687-708.

[12] M. IтоH, Weitzenböck formula for the Bach operator, to appear in Nagoya Math. $\mathrm{J}$.

[13] R. Kobayashi, Moduli of Einstein metrics on a K3 surface and degenerstion of type I, Advanced Studies in Pure Mathematics, Kähler Metrics and Moduli Spaces 18-II, Kinokuniya Publ., Tokyo, 1990, 257-311.

[14] R. Kobayashi And A. Todorov, Polarized period map for generalized K3 surfaces and the moduli of Einstein metrics, Tôhoku Math. J., 39 (1987), 341-363.

[15] S. Kobayashi, Transformation Groups in Differential Geometry, Springer-Verlag, Berlin-Heidelberg-New York, 1972.

[16] N. KoIso, Rigidity and stability of Einstein metrics. The case of compact symmetric spaces, Osaka J. Math., 17 (1980), 51-73. 
[17] M. KRECK, Isotopy classes of diffeomorphisms of $(k-1)$-connected almost parallelizable $2 k$-manifolds, Algebraic Topology Aahhus 1978, Lecture Notes in Math., 763, Springer-Verlag, 1979, 643-663.

[18] Y.T. SıU, Every K3 surface is Kähler, Invent. Math., 73 (1983), 139-150.

[19] C.T.C. WALL, Diffeomorphisms of 4-manifolds, J. London Math. Soc., 39 (1964), $131-140$.

[20] S. T. YAU, On the Ricci curvature of a compact Kähler manifold and the complex Monge-Ampère equation I, Com. Pure and Appl. Math., 31 (1978), 339-411.

INSTitute of Mathematics

UNIVERSITY OF TSUKUBA

305 JAPAN 